\title{
A cyclic fluctuation model for 24-h ambulatory blood pressure monitoring in Chinese patients with mild to moderate hypertension
}

\author{
Yu-cheng SHENG ${ }^{1}$, Kun WANG ${ }^{1,2}$, Ling XU ${ }^{1}$, Juan YANG ${ }^{1}$, Ying-chun $\mathrm{HE}^{1}$, Qing-shan ZHENG ${ }^{1,2, *}$ \\ ${ }^{1}$ Center for Drug Clinical Research, Shanghai University of Chinese Medicine, Shanghai 201203, China; ${ }^{2}$ Shanghai Municipal \\ Education Commission, E-Institute of Traditional Chinese Internal Medicine, Shanghai University of Traditional Chinese Medicine, \\ Shanghai 201203, China
}

Aim: The conventional method for analyzing 24-h ambulatory blood pressure monitoring (24-h ABPM) is insufficient to deal with the large amount of data collected. The aim of this study was to develop a novel cyclic fluctuation model for 24-h ABPM in Chinese patients with mild to moderate hypertension.

Methods: The data were obtained from 4 independent antihypertensive drug clinical trials in Chinese patients with mild to moderate hypertension. The measurements of 24-h ABPM at the end of the placebo run-in period in study 1 were used to develop the cyclic fluctuation model. After evaluated, the structural model was used to analyze the measurements in the other 3 studies. Models were fitted using NONMEM software.

Results: The cyclic fluctuation model, which consisted of 2 cosine functions with fixed-effect parameters for rhythm-adjusted 24-h mean blood pressure, amplitude and phase shift, successfully described the blood pressure measurements of study 1 . Model robustness was validated by the bootstrap method. The measurements in the other 3 studies were well described by the same structural model. Moreover, the parameters from all the 4 studies were very similar. Visual predictive checks demonstrated that the cyclic fluctuation model could predict the blood pressure fluctuations in the 4 studies.

Conclusion: The cyclic fluctuation model for 24-h ABPM deepens our understanding of blood pressure variability, which will be beneficial for drug development and individual therapy in hypertensive patients.

Keywords: blood pressure; 24-h ambulatory blood pressure monitoring; cyclic fluctuation model; NONMEM; hypertension; Chinese patients

Acta Pharmacologica Sinica (2013) 34: 1043-1051; doi: 10.1038/aps.2013.45; published online 17 Jun 2013

\section{Introduction}

Twenty-four-hour ambulatory blood pressure monitoring (24-h ABPM) is an important indicator in hypertension diagnosis and the evaluation of the effects of antihypertensives ${ }^{[1-3]}$. Multiple measurements of blood pressure within a day using 24-h ABPM can be relatively objective and can truly reflect the patient's blood pressure levels compared to casual blood pressure measurement. Thus, this method can diagnose white coat hypertension, masked hypertension and morning hypertension. Moreover, it also facilitates understanding the circadian rhythm of blood pressure and fluctuation characteristics. Recently, 24-h ABPM has been regarded as a confirmatory method for the diagnosis of hypertension in the British hyper-

\footnotetext{
* To whom correspondence should be addressed.

E-mail zhengqscn@21cn.com

Received 2012-08-28 Accepted 2013-03-14
}

tension guidelines ${ }^{[4]}$. In addition, 24-h ABPM is also indicated to predict target organ damage and the prognosis of cardiovascular disease patients. It has been confirmed that $24-\mathrm{h}$ average blood pressure is more related to target organ damage and the prognosis of hypertension than casual blood pressure measurement ${ }^{[5-7]}$.

The ideal antihypertensive drug should lead to a steady decline in blood pressure over an entire 24-h period. According to the guiding principle for clinical trials of new antihypertensive drugs by the Chinese SFDA, an ABPM study conducted in a certain number of hypertensive patients must be included as a part of new antihypertensive drug clinical trials $^{[8]}$. Although many 24-h ABPM studies have been conducted in China, the commonly used analytic method for 24-h ABPM is still insufficient with regard to the large amount of acquired data. Some cyclic fluctuation models for 24-h ABPM have been developed in non-Chinese populations ${ }^{[9,10]}$. How- 
ever, higher nighttime blood pressure has been observed in Chinese patients compared with non-Chinese patients ${ }^{[11,12]}$. In the Chinese population, a high-salt diet and gene polymorphisms may also influence the pattern of blood pressure changes $^{[13,14]}$. It is important to understand and describe the cyclic fluctuations of blood pressure in Chinese hypertensive patients. The goal of this study was to develop a cyclic fluctuation model for blood pressure in patients with mild to moderate hypertension in 4 antihypertensive drug clinical studies in China. This model will be beneficial for antihypertensive therapy in clinical practice and new drug development.

\section{Materials and methods}

\section{Data sources and study design}

The 24-h ABPM data were collected from 4 antihypertensive drug clinical trials that were conducted in accordance with the Declaration of Helsinki. Patients were enrolled in these 4 studies if they were 18-75 years of age with mild or moderate hypertension (mean sitting systolic $\mathrm{BP} /$ mean sitting diastolic BP, mild: 140-159/90-99 mmHg, moderate: 160-179/100-109 $\mathrm{mmHg})^{[15]}$. The major exclusion criteria included significant cardiovascular, hepatic, and renal disease and concomitant type 1 diabetes or uncontrolled type 2 diabetes, childbearing potential, current use of anticonvulsant or antidepressant therapy, and history of drug abuse. All subjects signed an informed consent before any study-related procedures were performed.

The 4 studies included a 2-week placebo run-in period, followed by an 8-week, open-label antihypertensive treatment period. Drugs (placebo or antihypertensive agents) were administered orally between 7 and 10 AM once daily. On study visit days, patients were instructed not to take their study medication until the assessments were completed. Twenty-four-hour ABPM was used at the end of each period. The blood pressure measurements in all studies were performed every $15 \mathrm{~min}$ from $8 \mathrm{AM}$ to $10 \mathrm{PM}$ and every $30 \mathrm{~min}$ from $10 \mathrm{PM}$ to $8 \mathrm{AM}$ with an Ambulatory Blood Pressure Monitor (SunTech Medical Instruments, Raleigh, NC, USA).
The blood pressure fluctuation model in this study was based on the 24-h ABPM data collected at the end of the run-in period. Patient demographics and the description of 24-h $\mathrm{ABPM}$ at the end of the placebo run-in period are shown in Table 1.

\section{Modeling and validation Structural model}

The blood pressure fluctuation model was based on a model of the 24-h ABPM profile without drugs present that was previously described by Hempel ${ }^{[9,10]}$. The original model for the ABPM measurements was represented as follows:

$$
B P_{\mathrm{k}}(t)=\text { Base }_{\mathrm{k}} \cdot\left\{1+\sum_{i=1}^{n} A_{\mathrm{ik}} \cdot \cos \left[\frac{\mathrm{i} \cdot \pi \cdot\left(t-P H S_{\mathrm{ik}}\right)}{0.5}\right]\right\}
$$

In this equation, $k=1$ is systolic blood pressure, $k=2$ is diastolic blood pressure, $\mathrm{BP}_{\mathrm{k}}(t)$ is the blood pressure as a function of $t$ (the unit of $t$ is day), Base $e_{\mathrm{k}}$ is the rhythm-adjusted 24-h mean, $\mathrm{A}_{\mathrm{ik}}$ is the amplitude of the cosine terms, and $\mathrm{PHS}_{\mathrm{ik}}$ is the parameter for phase shifts of the cosine terms. The number of cosine functions $(n)$ was selected based on the minimum objective function values (OFVs) and diagnosis plots. Additional forms of the original model were also tested.

Two independent sets of parameters were used to fit the fluctuation models for diastolic and systolic blood pressure measurements, respectively. Based on a graphical inspection of the raw data, equal values of phase-shift parameters (ie, $\mathrm{PHS}_{\mathrm{i} 1}=\mathrm{PHS}_{\mathrm{i} 2}$ ) for both diastolic and systolic blood pressure measurements were also used. Model selection was based on the goodness-of-fit plots, estimates and standard errors of the population parameters, and OFVs.

\section{Covariate model selection}

The influence of covariates was evaluated after the structural model was developed. The covariates considered in the BP model parameters were demographic factors [gender, age, weight, body mass index (BMI)]. Diagnostic plots of possible covariates versus the inter-individual variabilities were first

Table 1. Patient demographics and description of the data sets used.

\begin{tabular}{|c|c|c|c|c|}
\hline & Study 1 & Study 2 & Study 3 & Study 4 \\
\hline № of patients & 38 & 42 & 25 & 29 \\
\hline \multicolumn{5}{|l|}{ Sex } \\
\hline Male & 17 & 22 & 16 & 15 \\
\hline Female & 21 & 20 & 9 & 14 \\
\hline Age $(\text { year })^{*}$ & $51.6 \pm 7.83(35,69)$ & $50.9 \pm 7.91(35,69)$ & $51.0 \pm 12.3(28,72)$ & $48.8 \pm 9.79(36,72)$ \\
\hline $\operatorname{BMI}\left(\mathrm{kg} / \mathrm{m}^{2}\right)^{*}$ & $24.0 \pm 2.91(17.6,28.7)$ & $26.2 \pm 2.80(20.8,30.0)$ & $24.5 \pm 2.72(18.4,29.7)$ & $26.0 \pm 2.75(23.0,30.3)$ \\
\hline Average 24-h SBP $(\mathrm{mmHg})^{*}$ & $141 \pm 15.6(120,175)$ & $145 \pm 16.1(122,194)$ & $139 \pm 15.1(125,165)$ & $137 \pm 13.9(118,157)$ \\
\hline Average 24-h DBP $(\mathrm{mmHg})^{*}$ & $90.4 \pm 10.2(80,119)$ & $92.8 \pm 10.8(80,124)$ & $90.2 \pm 10.5(80,117)$ & $90.2 \pm 9.8(81,103)$ \\
\hline № of SBP measurements & 2110 & 2927 & 2346 & 1687 \\
\hline № of DBP measurements & 2110 & 2927 & 2346 & 1687 \\
\hline
\end{tabular}

\footnotetext{
${ }^{*}$ Mean \pm SD (minimum and maximum values in parentheses). Average 24-h blood pressures were directly read from the automatic analysis.
} 
used to screen for relevant covariates that could influence the individual parameters obtained from the structural model. The screened covariates were evaluated by changes of the OFV. Assuming that the differences in the OFVs had a chisquare distribution, a decrease in OFV greater than 3.84 from the structural model $(P<0.05)$ was used for stepwise forward inclusion. The full model was built by introducing all significant covariates. Subsequently, the final model was developed using a stepwise backward elimination procedure. Covariates remained in the final model when the elimination of the variable caused a significant increase in OFV greater than 6.63 compared to the full model $(P<0.01)$. Goodness-of-fit graphs were also applied for model evaluation.

Continuous covariates were standardized to their mean values and were modeled as an exponential function by the following equation:

$$
\theta=\theta_{\mathrm{T}}\left(\frac{\operatorname{cov}}{\text { Mean }_{\mathrm{cov}}}\right)^{\theta \mathrm{cov}}
$$

Categorical variables were modeled using a proportional change model as shown:

$$
\theta=\theta_{\mathrm{T}}\left(\operatorname{cov}_{1}+\theta_{\text {cov }} \operatorname{cov}_{2}\right)
$$

where $\operatorname{cov}_{1}$ and $\operatorname{cov}_{2}$ were indicator variables set equal to ' 1 ' to denote the ith covariate value and ' 0 ' otherwise, $\theta_{\mathrm{T}}$ was the model parameter for $\operatorname{cov}_{1}$, and $\theta \operatorname{cov}$ was the fractional change of $\operatorname{cov}_{2}$ from $\theta_{\mathrm{T}}$, respectively.

\section{Statistical models}

We attempted to add the inter-individual variability $\left(\eta_{i}\right)$ into the parameters of each model, and it was assumed to be log-normally distributed. The expression for subject $i$ is $P_{\mathrm{i}}=P_{\mathrm{tv}}{ }^{*} \exp \left(\eta_{\mathrm{i}}\right)$, where $P_{\mathrm{i}}$ is the estimate for the model parameter of the $\mathrm{i}$ individual, $P_{\mathrm{tv}}$ is the typical population estimate, and $\eta_{i}$ is the inter-individual random-effect assumed to have an expected mean 0 and variance $\omega_{i}^{2}$.

The residual variability was modeled with an additive error model as shown: $Y_{\mathrm{ij}}=\mathrm{IPRE}_{\mathrm{ij}}+\varepsilon$, where $Y_{\mathrm{ij}}$ and IPRE $\mathrm{ij}_{\mathrm{ij}}$ represent the jth observed or predicted blood pressure for the ith subject, respectively. $\varepsilon$ is the residual random effect defined as being normally distributed with mean 0 and variance $\sigma^{2}$.

\section{Software}

The population analysis was performed with non-linear mixed effects modeling using NONMEM version 7.2 (Icon Development Solutions, Ellicott City, MD, USA). NONMEM runs were executed using Wings for NONMEM (WFN720, http:/ / wfn.sourceforge.net). The first-order conditional estimation method with interaction (FOCE-I) was used throughout the analysis. Perl-speaks-NONMEM 3.5.3 ${ }^{[16]}$ was used to facilitate processing the NONMEM output and for parallel execution of the bootstrap. Xpose ${ }^{[17]}$ was used to evaluate the goodness-offit of the models and for plotting.

\section{Model evaluation}

Graphical and non-parametric statistical methods were used for model evaluation. Goodness-of-fit plots, including plots of observed versus population and versus individual predicted values, individual weighted residuals (IWRES) versus individual predicted values and weighted residuals (WRES) versus time, were used to evaluate model bias. The final model was evaluated using a nonparametric bootstrap re-sampling technique. A total of 1000 bootstrap replicate datasets were generated by sampling randomly from the original data of study 1 with replacement. The accuracy of the model parameters was evaluated by comparing the $90 \%$ confidence interval of the parameter estimates from the bootstrap datasets with the parameter values obtained from the original dataset.

The predictive performance of the final model was validated internally using the data from study 1 . A visual predictive check (VPC) based on 1000 simulations for the final model was used to evaluate the model. The measured time points were binned into one-hour intervals. For each bin, the median blood pressure and the 2.5th and 97.5th percentiles were calculated from the simulated data. The observed blood pressure and confidence interval of the simulated data were plotted together for visual inspection.

The data from study 1 were used as the developmental dataset for model building. After model evaluation and validation, the same structural model was used for the data from the remaining 3 studies. The ability of the final model to describe the observed data was also investigated using the visual predictive check. For generalization to future studies, the data from all 4 studies were also combined and analyzed using the structural model built in study 1 . The same model-developing procedure introduced previously was also applied for the pooled data.

\section{Results}

The following equation containing two cosine functions was selected as the circadian rhythm model to describe the blood pressure changes over time:

$$
B P_{\mathrm{k}}(t)=\text { Base }_{\mathrm{k}}+A_{\mathrm{lk}} \cdot \cos \left[\frac{2 \cdot \Pi \cdot\left(t-P H S_{1}\right)}{0.5}\right]+A_{2 \mathrm{k}} \cdot \cos \left[\frac{\Pi \cdot\left(t-P H S_{2}\right)}{0.5}\right]
$$

In this equation, $\mathrm{k}=1$ is systolic blood pressure, $\mathrm{k}=2$ is diastolic blood pressure, $\mathrm{BP}_{\mathrm{k}}(t)$ is the blood pressure as a function of $t$ (the unit of $t$ is day), Base $e_{k}$ is the rhythm-adjusted 24-h mean, $\mathrm{A}_{1 \mathrm{k}}$ and $\mathrm{A}_{2 \mathrm{k}}$ are the amplitude of the cosine terms, and $\mathrm{PHS}_{1}$ and $\mathrm{PHS}_{2}$ are the parameters for phase shifts of the cosine terms.

As shown in the plots of systolic and diastolic blood pressure versus time, the time trends of both blood pressure measurements were similar. The circadian rhythm models were developed successfully for diastolic and systolic blood pressure measurements, respectively (Figure 1). Because the phase-shift parameters in the two models were very close, the same phase-shift parameters were used for the final model in which systolic and diastolic blood pressure were modeled together. The parameter estimates and their corresponding standard error (in percentage points) obtained from the final model are listed in Table 2. The typical values of the rhythm- 
Table 2. Model parameter estimates of the diastolic and systolic 24-h ABPM with 1000 nonparametric bootstrap procedures of Study 1.

\begin{tabular}{|c|c|c|c|c|c|}
\hline Parameter & Annotations & $\begin{array}{l}\text { Estimate } \\
\text { (SEM\%) }\end{array}$ & $\begin{array}{l}\text { Shrinkage\% } \\
\text { ( } P \text { value })\end{array}$ & $\begin{array}{l}\text { Bootstrap } \\
\text { median }\end{array}$ & $\begin{array}{c}\text { Bootstrap } \\
95 \% \mathrm{Cl}\end{array}$ \\
\hline \multicolumn{6}{|c|}{ Cyclic fluctuations model parameters } \\
\hline Base $_{1}$ & Rhythm-adjusted 24-h mean SBP (mmHg) & $140(1.8)$ & & 140 & $136-145$ \\
\hline Base $_{2}$ & Rhythm-adjusted 24-h mean DBP (mmHg) & $89.5(1.8)$ & & 89.6 & $86.7-92.6$ \\
\hline $\mathrm{A}_{11}$ & Amplitude first cosine term of SBP & $7.52(8.7)$ & & 7.52 & $6.37-8.79$ \\
\hline$A_{21}$ & Amplitude second cosine term of SBP & $8.64(9.1)$ & & 8.58 & $6.89-10.19$ \\
\hline $\mathrm{A}_{12}$ & Amplitude first cosine term of DBP & $5.61(7.6)$ & & 5.60 & $4.70-6.50$ \\
\hline $\mathrm{A}_{22}$ & Amplitude second cosine term of DBP & $6.27(9.3)$ & & 6.32 & $5.21-7.50$ \\
\hline $\mathrm{PHS}_{1}$ & Phase-shift first cosine term & $-0.652(1.3)$ & & -0.651 & $-0.668-0.862$ \\
\hline $\mathrm{PHS}_{2}$ & Phase-shift second cosine term & $3.61(0.8)$ & & 3.61 & $3.50-3.69$ \\
\hline \multicolumn{6}{|c|}{ Inter-individual variability } \\
\hline$\omega_{\text {Base } 1}(\mathrm{CV} \%)$ & Interindividual variability of Base $_{1}$ & $0.079(20.6)$ & $-0.68(0.999)$ & 0.079 & $0.061-0.094$ \\
\hline$\omega_{\text {Base2 }}(\mathrm{CV} \%)$ & Interindividual variability of $\mathrm{Base}_{2}$ & $0.12(21.3)$ & $-0.48(0.999)$ & 0.12 & $0.094-0.146$ \\
\hline$\omega_{\mathrm{A} 11}(\mathrm{CV} \%)$ & Interindividual variability of $A_{11}$ & $5.63(31.8)$ & $20.5(0.232)$ & 5.47 & $3.42-7.14$ \\
\hline$\omega_{\mathrm{A} 21}(\mathrm{CV} \%)$ & Interindividual variability of $A_{21}$ & $4.73(33.0)$ & $17.9(0.282)$ & 4.74 & $3.12-6.91$ \\
\hline$\omega_{\mathrm{A} 12}(\mathrm{CV} \%)$ & Interindividual variability of $A_{12}$ & $6.58(37.6)$ & $21.6(0.234)$ & 6.39 & $3.20-8.70$ \\
\hline$\omega_{\mathrm{A} 22}(\mathrm{CV} \%)$ & Interindividual variability of $A_{22}$ & $6.71(35.6)$ & $16.6(0.421)$ & 6.36 & $4.05-8.83$ \\
\hline$\omega_{\text {PHS1 }}(\mathrm{CV} \%)$ & Interindividual variability of $\mathrm{PHS}_{1}$ & $10.9(27.5)$ & $4.96(0.933)$ & 10.05 & $7.0-13.3$ \\
\hline$\omega_{\text {PHS2 }}(\mathrm{CV} \%)$ & Interindividual variability of $\mathrm{PHS}_{2}$ & $1.24(37.6)$ & $0.23(0.988)$ & 1.17 & $0.59-1.6$ \\
\hline \multicolumn{6}{|c|}{ Residual variability } \\
\hline$\sigma_{\mathrm{SBP}}$ & Intraindividual residual variability of SBP ( $\mathrm{mmHg}$ ) & $12.85(6.5)$ & 2.93 & 12.9 & $12.1-13.7$ \\
\hline$\sigma_{\mathrm{DBP}}$ & Intraindividual residual variability of DBP $(\mathrm{mmHg})$ & $9.11(7.9)$ & 2.96 & 9.10 & $8.46-9.79$ \\
\hline
\end{tabular}

SEM\%, standard error \%; 95\% Cl, 95\% confidence interval; CV\%, coefficient of variation; SBP, systolic blood pressure; DBP, diastolic blood pressure; 24-h ABPM, 24-h ambulatory blood pressure monitoring.

adjusted 24-h mean diastolic and systolic blood pressures were $140 \mathrm{mmHg}$ and $89.5 \mathrm{mmHg}$, respectively. The standard error percentages of all fixed-effect parameters were less than $10 \%$, indicating that these parameters were sufficiently accurate and that the blood pressures were well described by the final model. The goodness-of-fit plots (Figure 1) of the predicted and observed data indicated that the model adequately described the systolic and diastolic blood pressures over the entire 24-h period. The weighted residuals were evenly distributed around zero, and most of these were within an SD of \pm 2 of the normalized units. Individual plots (Figure 2) of observations, individual predictions and population predictions versus time also indicated the model's adequate capture of both systolic and diastolic blood pressure measurements. The covariates, including gender, age, weight and BMI, did not significantly increase the goodness of fit.

Of the 1000 bootstrap re-sampling datasets, 974 were minimized successfully with the covariance step. Table 2 presents the results of the bootstrap distributions for the parameters of each model. The medians of the bootstrap re-samples of all parameters were similar to the estimations based on the original dataset. In addition, the relatively small bootstrapped confidence interval of all parameters indicated the accuracy and precision of the final model.

A total of 1000 simulated datasets based on the model parameters and graphical forecasts were also evaluated. As shown in the visual predictive checks (Figure 3, Study 1) based on the estimations of the final model, the model prediction and the observed measurements of the systolic and diastolic blood pressures were in good agreement. This also implied that the final model satisfyingly captured the tendency of the 24-h blood pressure fluctuations.

For the 24-h blood pressure measurements in Studies 2, 3 and 4 , the predictions of the same structural model were good. All parameter estimates were similar among the 4 studies (Table 3). The visual predictive check of these studies (Figure 3 , Studies 2, 3, and 4) also illustrated that the circadian rhythm model could adequately describe 24-h blood pressure.

When the data of the 24-h blood pressure measurements were pooled from all 4 studies, the fluctuation model also performed well, and only slight differences were shown in the parameters between the pooled analysis and the 4 study analyses. This indicated that the developed circadian rhythm model was robust and predictable. The inter-individual variability versus study plot and the residual variability versus study plot implied that there were no remarkable differences in the parameters among studies. OFVs were not decreased significantly when inter-study variabilities were introduced into the model. No covariate was introduced as a significant factor into the pooled model. 
Basic goodness-of-fit plots (SBP)
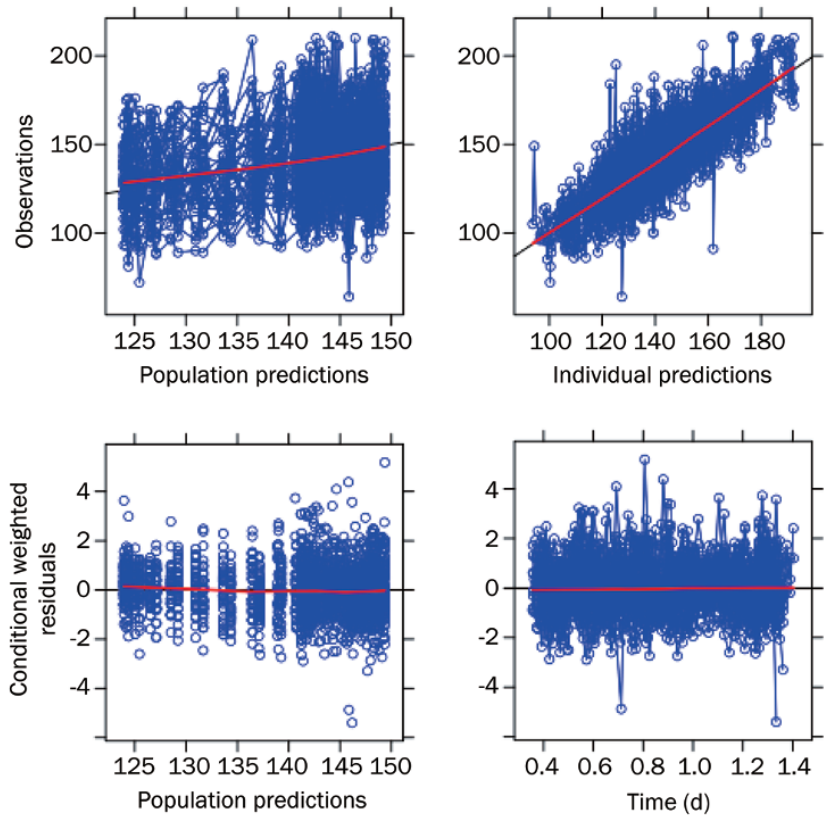

Basic goodness-of-fit plots (SBP)

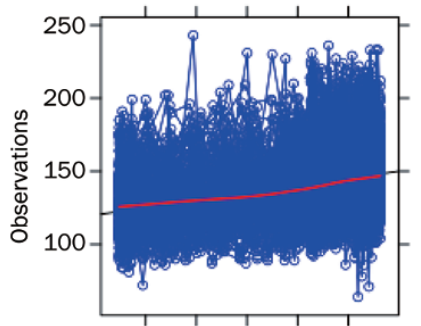

125130135140145 Population predictions

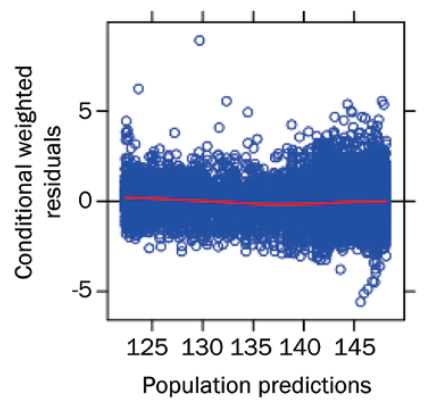

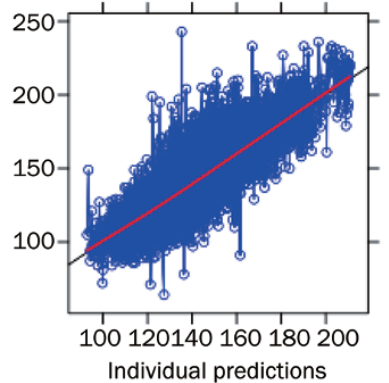

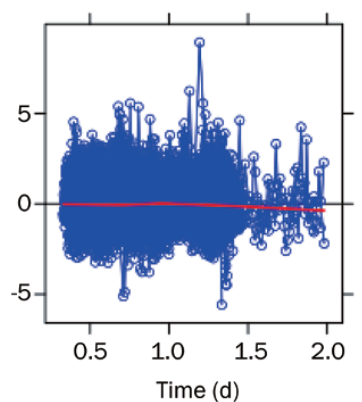

Basic goodness-of-fit plots (DBP)
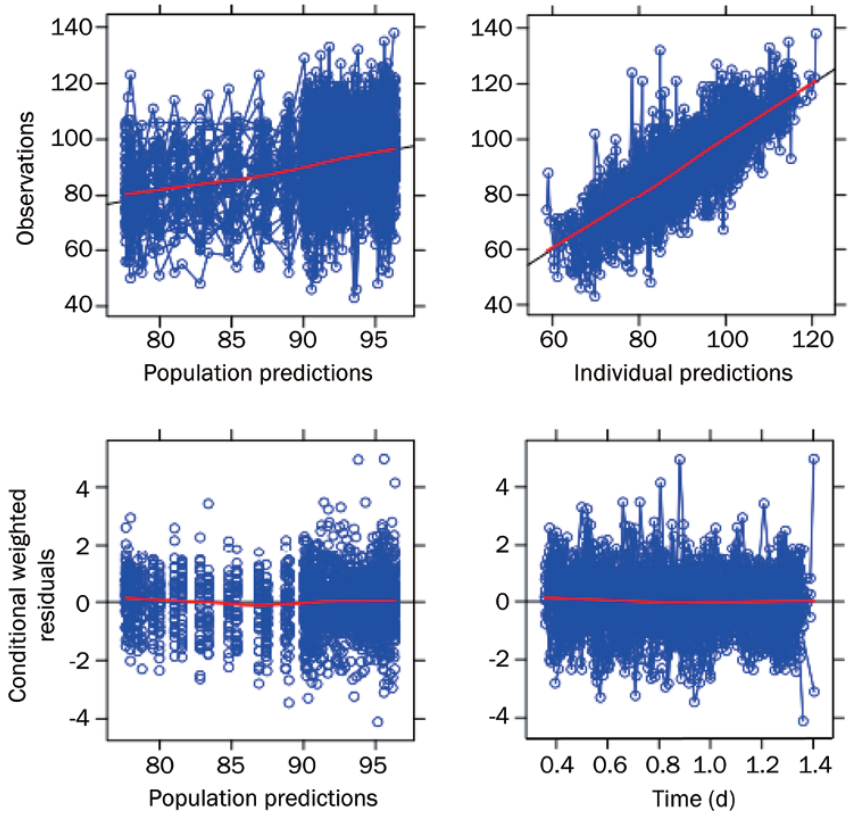

Basic goodness-of-fit plots (DBP)
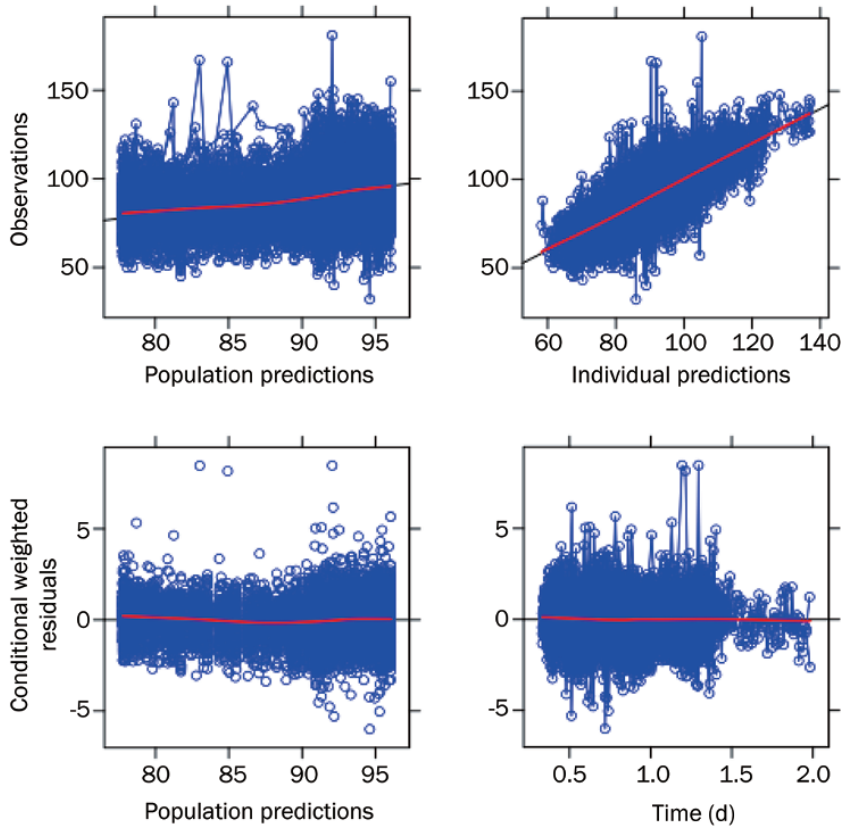

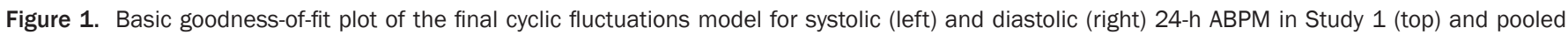
analysis (bottom).

\section{Discussion}

Compared to casual blood pressure measurements, more data points are measured by 24-h ABPM. Thus, 24-h ABPM provides a more precise diagnosis and better prediction of clinical outcomes in patients with hypertension and cardiovascular diseases. However, the indices of conventional analyses of 24-h ABPM, such as the trough-to-peak ratio, smoothness index, average 24-h BP and average daytime and nighttime
BP, do not take full advantage of the features of large amounts of data. Population modeling methods can effectively use all measurements from 24-h ABPM and reduce the influence of different measurement schedules among subjects.

The circadian rhythm model was successfully applied in this study to capture the cyclic fluctuations of both systolic and diastolic blood pressures within $24 \mathrm{~h}$. The rhythm-adjusted 24-h mean values of SBP and DBP from the model were almost 

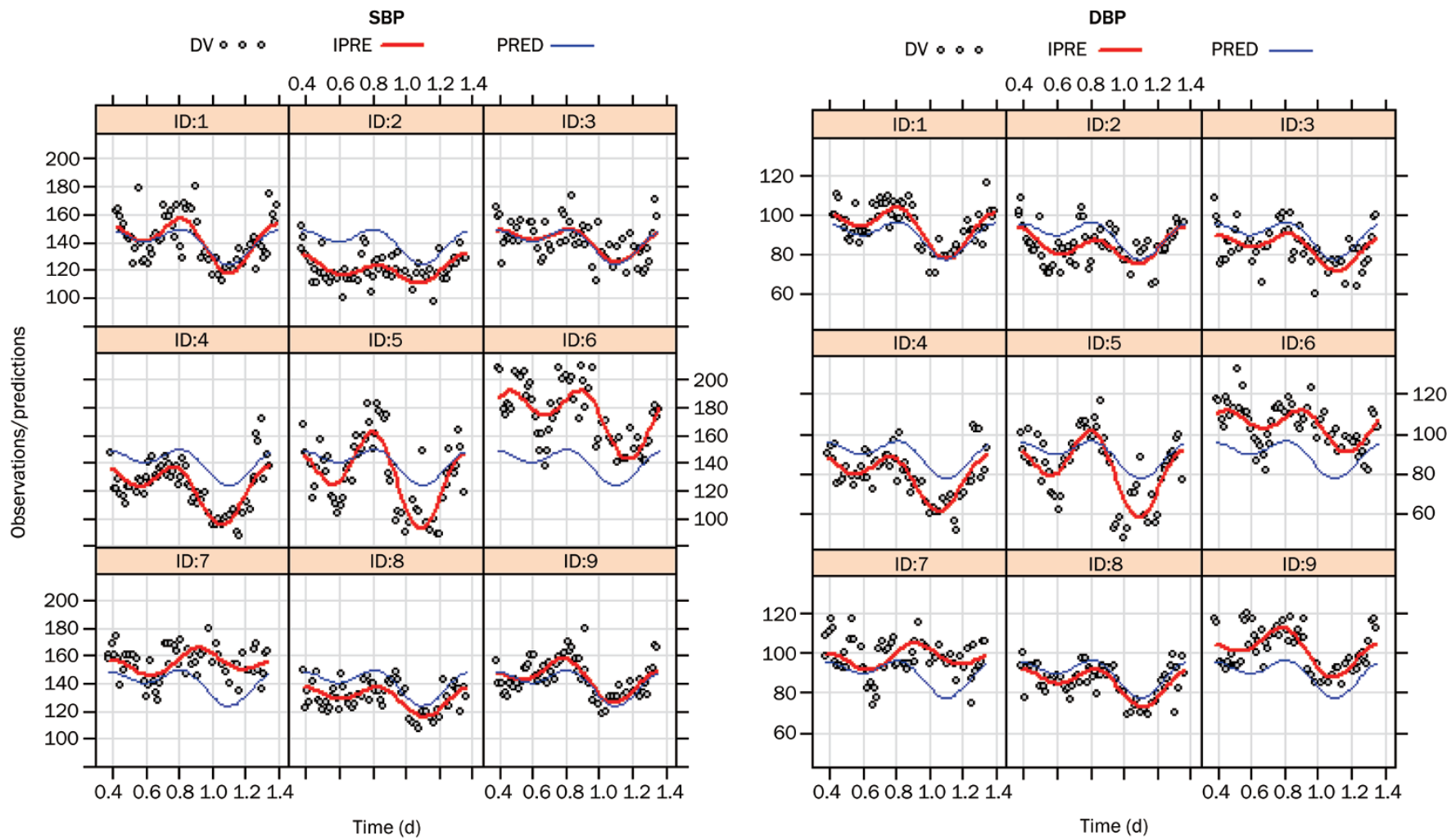

Figure 2. Individual model prediction of systolic (left) and diastolic (right) 24-h ABPM. Only the plots of the first 9 subjects were shown.

Table 3. Cyclic fluctuations model parameter estimates of the diastolic and systolic 24-h ABPM in Study 2, 3, and 4.

\begin{tabular}{|c|c|c|c|c|}
\hline Parameter & $\begin{array}{c}\text { Study } 2 \\
\text { Estimate (SEM\%) }\end{array}$ & $\begin{array}{c}\text { Study } 3 \\
\text { Estimate (SEM\%) }\end{array}$ & $\begin{array}{c}\text { Study } 4 \\
\text { Estimate (SEM\%) }\end{array}$ & $\begin{array}{c}\text { Overall }^{*} \\
\text { Estimate (SEM\%) }\end{array}$ \\
\hline \multicolumn{5}{|c|}{ Cyclic fluctuations model parameters } \\
\hline Base $_{2}$ & $91.7(1.8)$ & $88.8(1.2)$ & $88.5(0.8)$ & $89.5(0.7)$ \\
\hline$A_{11}$ & $7.83(8.5)$ & $6.07(8.6)$ & $7.11(9.2)$ & $7.2(4.6)$ \\
\hline $\mathrm{A}_{22}$ & $6.39(10.1)$ & $6.05(9.8)$ & $7.16(7.0)$ & $6.55(5.1)$ \\
\hline $\mathrm{PHS}_{1}$ & $-2.18(0.6)$ & $-2.16(0.3)$ & $0.84(1.1)$ & $-2.16(0.2)$ \\
\hline $\mathrm{PHS}_{2}$ & $3.61(0.7)$ & $3.58(0.4)$ & $4.58(0.4)$ & $3.59(0.3)$ \\
\hline \multicolumn{5}{|c|}{ Inter-individual variability } \\
\hline$\omega_{\text {Base1 }}$ & $0.108(26.2)$ & $0.068(23)$ & $0.0728(16.6)$ & $0.0933(13.4)$ \\
\hline$\omega_{\mathrm{A} 12}$ & $0.327(34.5)$ & $0.523(29.7)$ & $0.506(24.9)$ & $0.439(16.2)$ \\
\hline$\omega_{\mathrm{A} 22}$ & $0.547(33)$ & $0.660(22.7)$ & $0.415(22.0)$ & $0.502(14.3)$ \\
\hline$\omega_{\text {PHS1 }}$ & $0.0379(25.6)$ & $0.0128(28.1)$ & $0.0816(23.3)$ & $0.0296(15.5)$ \\
\hline$\omega_{\mathrm{PHS} 2}$ & $0.0424(29.5)$ & $0.0182(52.6)$ & $0.0318(31.7)$ & $0.0390(19.7)$ \\
\hline \multicolumn{5}{|c|}{ Random variability } \\
\hline$\sigma_{\mathrm{SBP}}$ & $12.8(6.7)$ & $12.6(6.9)$ & $11.3(5.9)$ & $12.2(3.4)$ \\
\hline$\sigma_{\mathrm{DBP}}$ & $9.28(6.3)$ & $9.74(10)$ & $8.96(5.6)$ & $9.23(3.7)$ \\
\hline
\end{tabular}

SEM\%, standard error \%; *Combined data of Study 2, 3, and 4. 
A

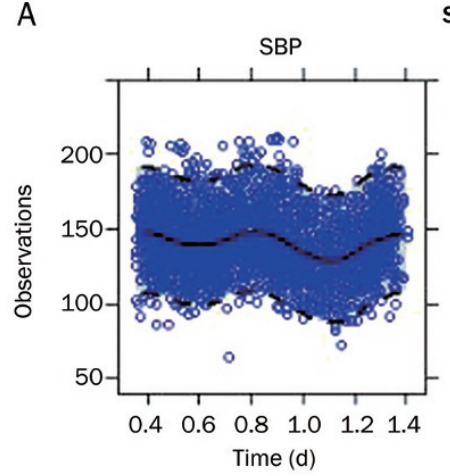

Study 1

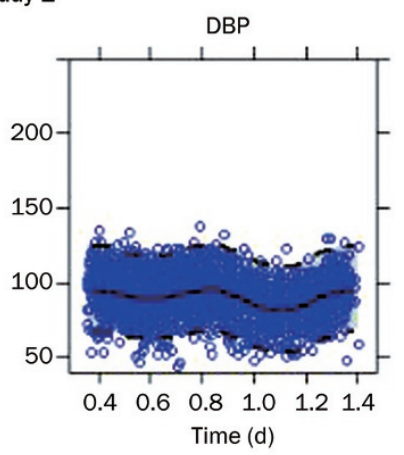

Study 3
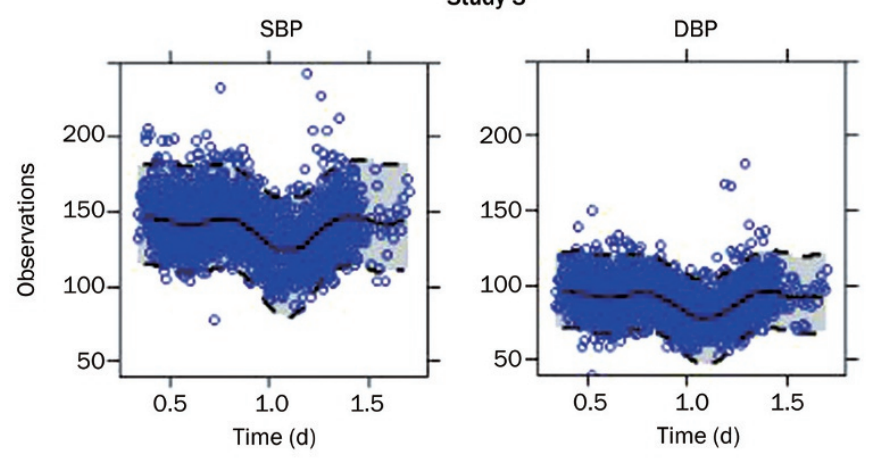

B

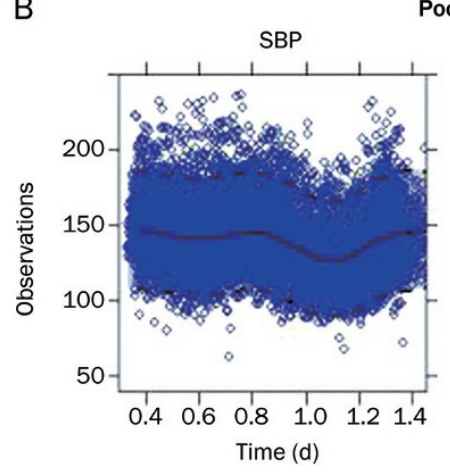

Pooled analysis

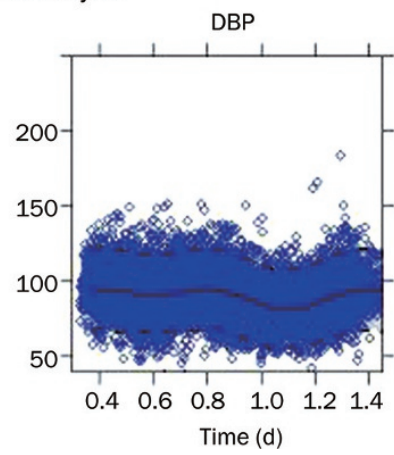

equivalent to the averaged values of those from the raw data. Compared with the published models for 24-h ABPM, which were studied in Europe ${ }^{[9,10]}$, rhythm-adjusted 24-h mean BPs (including SBP and DBP) were very close to those in Reference $^{[10]}$ but were slightly less than in that in Reference ${ }^{[9]}$. The likely reason is that only patients with mild-to-moderate hypertension were enrolled in our studies and in Reference ${ }^{[10]}$, and no such criteria were mentioned in Reference ${ }^{[9]}$. In addition, the additive residual variabilities for both SBP and DBP were similar to those of previous models. However, there were some features of the circadian rhythm model in this study. First, the model structure changed from multiplication to addition based on goodness of fit. Second, the same phaseshift parameters were applied to describe both systolic and diastolic blood pressures in the model. Because the model development procedure was based on previous experience, these modifications most likely indicate that the cyclic pattern

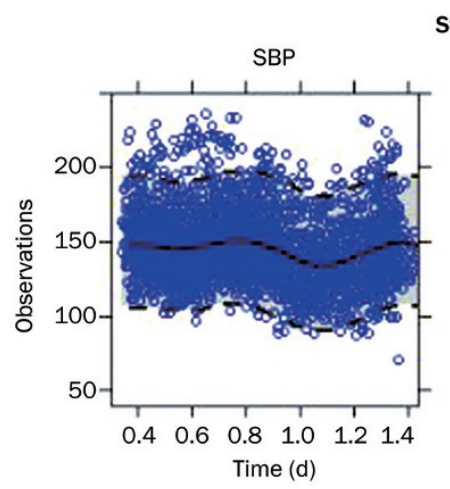

Study 2
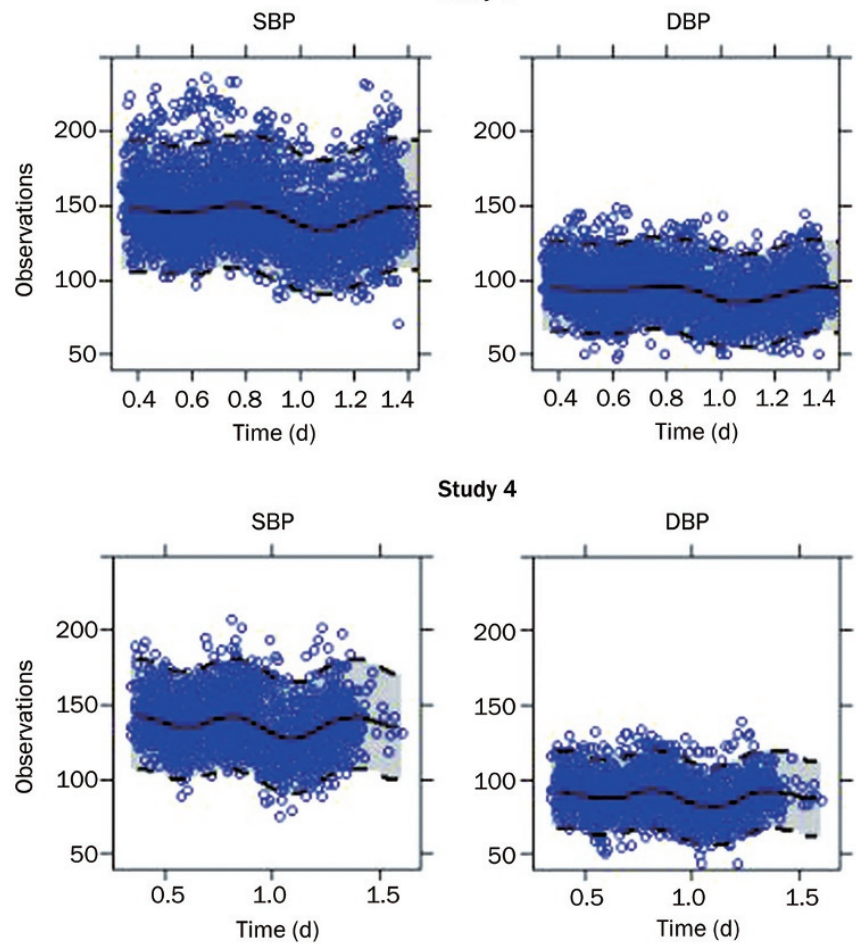

Study 4

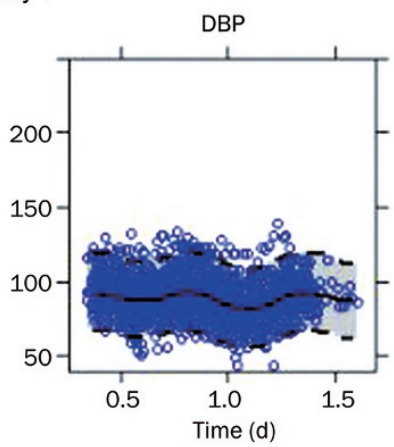

Figure 3. Visual predictive check of the cyclic fluctuations model for four 24-h ABPM studies and pooled analysis. The open circle represents the observations, the lines represent 5th, 50th, and 95th percentiles of the 1000 simulated datasets.

of blood pressure fluctuations in Chinese patients is slightly different from that in non-Chinese patients.

The predictive performance of the model was assessed in two steps. The first step was performed using a visual predictive check to evaluate the ability of the model to generate the data on which the model was built (Study 1). The second step involved 3 datasets from three other 24-h ABPM studies (Studies 2-4). In both steps, the model performed well and showed the ability to predict the central tendency of blood pressure in patients with mild to moderate hypertension in China with reasonable confidence. Furthermore, the fluctuating changes of blood pressure showed a "two peaks and a valley" feature in the visual predictive check plots. The "dipping pattern," which indicated that the average difference between daytime and nighttime systolic and diastolic BPs was $10 \%-20 \%$, was also observed as an overall trend of all the studies. Although "non-dipper" changes (nocturnal decline of $<10 \%$ ) were found 
in some patients (IDs 2 and 7 in Figure 2), the model's predictive performance in these subjects was good as well. This pattern in the patients is also an important source of interindividual variability of the model parameters. Eating, physical activity and emotional state, which will affect blood pressure readings, were not recorded in these 4 studies and were therefore not included in the model. These influential factors should be collected in future 24-h ABPM studies and introduced into the model in an appropriate way to improve the accuracy of model predictions.

According to the regular circadian rhythm pattern of blood pressure fluctuations, individualized therapy based on the principle of time therapeutics has gradually received much more attention in the treatment of essential hypertension ${ }^{[18]}$. The cyclic fluctuation model developed in this study can be applied to estimate the variation in individual hypertensive patients. It also can provide the basis for individualized treatment, such as selecting the appropriate antihypertensive drugs and adjusting the dose and the time that medicine is taken.

In this study, the cyclic fluctuation model, which was built based on the data from study 1 , was still able to achieve successful performance in fitting and predicting data from the other three studies. The general parameters were also obtained from the pooled data of four studies. The criteria for screening patients with mild-to-moderate hypertension in our studies also suggest the possibility of using this model for other antihypertensive studies. Moreover, the model parameters of all studies were very similar, indicating that this model has good productivity and extensive suitability and could be used as a reference in future 24-h ABPM studies. Blood pressure can be described and simulated at any time point using the developed model. In new antihypertensive drug development, the pharmacokinetic-pharmacodynamic model can be added to the circadian rhythm model to describe and predict blood pressures over time after drug administration. Using these models, different dosing regimens and study designs of new antihypertensive clinical trials can be simulated and assessed $^{[19]}$. As a result, the uncertainty of new antihypertensive drug development could be reduced by answering a series of "what-if" problems based on these models.

\section{Acknowledgements}

This study was supported by the National Key Technology R\&D Program Project (2008BAI51B03), the National Key Project for IND (No 2012ZX09303-003 and 2012ZX09303009-001), and the Leading Academic Discipline Project of Shanghai Municipal Education Commission (№ 085ZY1202, J50303, SZY11059, and ZYSNXD-CC-LCPJ). We also thank Dr Guangli MA and Dr Jun-jie DING for providing guidance during the modeling process. The authors would like to thank Beijing Chao-Yang Hospital, Beijing Friendship Hospital, Beijing Xuanwu Hospital and Baotou Central Hospital for collecting the 24-h ABPM data.

\section{Author contribution}

Yu-cheng SHENG designed the research and wrote the paper;
Qing-shan ZHENG revised the paper; Yu-cheng SHENG, Juan YANG, and Ying-chun HE analyzed the data and plotted the figures; Kun WANG and Ling XU provided technical assistance.

\section{References}

1 Kanbay M, Turkmen K, Ecder T, Covic A. Ambulatory blood pressure monitoring: from old concepts to novel insights. Int Urol Nephrol 2012; 44: 173-82.

21999 World health organization-international society of hypertension guidelines for the management of hypertension. Guidelines subcommittee. J Hypertens 1999; 17: 151-83.

3 Chobanian AV, Bakris GL, Black HR, Cushman WC, Green LA, Izzo JL Jr, et al. The seventh report of the joint national committee on revention, detection, evaluation, and treatment of high blood pressure: the JNC 7 report. JAMA 2003; 289: 2560-72.

4 National Institution for Health and Clinical Excellence (NICE). Hypertension: the clinical management of primary hypertension in adults, 2011 Aug.[cited 2012 June 12]. Available from: http://www. nice.org.uk/nicemedia/live/13561/56008/56008.pdf.

5 Perloff D, Sokolow M. Ambulatory blood pressure measurements, prognostic implications. Archives des maladies du coeur et des vaisseaux 1991; 84: 21-7.

6 Bliziotis IA, Destounis A, Stergiou GS. Home versus ambulatory and office blood pressure in predicting target organ damage in hypertension: a systematic review and meta-analysis. J Hypertens 2012; 30: 1289-99.

7 Hansen TW, Kikuya M, Thijs L, Bjorklund-Bodegard K, Kuznetsova T, Ohkubo T, et al. Prognostic superiority of daytime ambulatory over conventional blood pressure in four populations: a meta-analysis of 7030 individuals. J Hypertens 2007; 25: 1554-64.

8 Center for Drug Evaluation S. Guidance for clinical trials of new antihypertensive drugs. Beijing: [updated 2007 Aug 14; cited 2012 Aug 28]. Available from: www.cde.org.cn/attachmentout. do?mothed=list\&id=378.

9 Hempel G, Karlsson MO, de Alwis DP, Toublanc N, McNay J, Schaefer HG. Population pharmacokinetic-pharmacodynamic modeling of moxonidine using 24-h ambulatory blood pressure measurements. Clin Pharmacol Ther 1998; 64: 622-35.

10 Troconiz IF, de Alwis DP, Tillmann C, Callies S, Mitchell M, Schaefer HG. Comparison of manual versus ambulatory blood pressure measurements with pharmacokinetic-pharmacodynamic modeling of antihypertensive compounds: application to moxonidine. Clin Pharmacol Ther 2000; 68: 18-27.

11 Li Y, Staessen JA, Lu L, Li LH, Wang GL, Wang JG. Is isolated nocturnal hypertension a novel clinical entity? Findings from a Chinese population study. Hypertension 2007; 50: 333-9.

12 Li Y, Wang JG, Gao P, Guo H, Nawrot T, Wang G, et al. Are published characteristics of the ambulatory blood pressure generalizable to rural Chinese? The JingNing population study. Blood Pressure Monitoring 2005; 10: 125-34.

13 Kelly TN, Rice TK, Gu D, Hixson JE, Chen J, Liu D, et al. Novel genetic variants in the alpha-adducin and guanine nucleotide binding protein beta-polypeptide 3 genes and salt sensitivity of blood pressure. Am J Hypertens 2009; 22: 985-92.

14 Li YY. alpha-Adducin Gly460Trp gene mutation and essential hypertension in a Chinese population: a meta-analysis including 10,960 subjects. PloS one 2012; 7: e30214.

15 Mancia G, De Backer G, Dominiczak A, Cifkova R, Fagard R, Germano G, et al. 2007 Guidelines for the management of arterial hypertension: the task force for the management of arterial hypertension of the 
European society of hypertension (ESH) and of the European society of cardiology (ESC). J Hypertens 2007; 25: 1105-87.

16 Lindbom L, Ribbing J, Jonsson EN. Perl-speaks-NONMEM (PsN) a Perl module for NONMEM related programming. Comp Methods Program Biomed 2004; 75: 85-94.

17 Jonsson EN, Karlsson MO. Xpose - an S-PLUS based population pharmacokinetic/pharmacodynamic model building aid for NONMEM. Comp Methods Program Biomed 1999; 58: 51-64.
18 Hermida RC, Ayala DE, Chayan L, Mojon A, Fernandez JR. Administration-time-dependent effects of olmesartan on the ambulatory blood pressure of essential hypertension patients. Chronobiol Int 2009; 26: 61-79.

19 Lalonde RL, Kowalski KG, Hutmacher MM, Ewy W, Nichols DJ, Milligan PA, et al. Model-based drug development. Clin Pharmacol Ther 2007; 82: 21-32. 